\title{
HARDWARE DE BAIXO CUSTO PARA O ENSINO DA SINTONIA DE CONTROLADORES
}

\author{
R. D. PEREIRA FILHO \\ Universidade Federal do Rio Grande - FURG \\ renatodutrapereira@gmail.com
}

\begin{abstract}
RESUMO - O ensino de controle de processos químicos aborda, entre outros aspectos, a identificação de modelos dinâmicos de processos, a definição de estratégias e a sintonia de controladores. Pacotes como Matlab e o Scilab, bem como simuladores comerciais, são usados no ensino, mas a dinâmica do processo a ser analisado pode estar explícita e visível ao usuário da aplicação computacional, o que pode ser indesejável, bem como pode ser necessário o desembolso com licenças de software. Este trabalho teve como objetivos projetar, prototipar, programar e implementar um hardware para emular processos baseados na dinâmica de primeira ordem, operando em malha aberta e/ou malha fechada com um controlador $\mathrm{P}+\mathrm{I}+\mathrm{D}$. O hardware foi centrado em um Arduino Nano, e conta com painel LCD, além da interface serial de entrada/saída para a interação com os usuários. O programa simulador dinâmico foi desenvolvido em $\mathrm{C}++$, para a solução do problema do valor inicial da EDO de primeira ordem, pelo método de Euler, e reside no microcontrolador ATmega328. O tratamento dos resultados das simulações em malha aberta, para identificação do processo desconhecido e a sintonia dos parâmetros do controlador feedback com lei $\mathrm{P}+\mathrm{I}+\mathrm{D}$ são executados off-line pelos usuários, enquanto o teste do desempenho em malha fechada é feito no hardware desenvolvido. O protótipo foi usado na disciplina de Controle de Processos Químicos da FURG, permitindo uma maior familiarização dos estudantes com o projeto e sintonia de controladores PID.
\end{abstract}

\section{INTRODUÇÃO}

O uso de softwares de simulação, comerciais ou não, dentre os quais estão Aspen Plus, Aspen Hysys, e EMSO, é disseminado no ensino, na pesquisa e no dia-a-dia do profissional de engenharia química. Já o uso de pacotes matemáticos proprietários como Matlab, Mathematica, MathCad, ou "open source" como o Scilab e o Octave, bem como linguagens de programação, como o C ou o Python, é bem mais comum no meio acadêmico do que no meio industrial. Todas essas ferramentas, em conjunto ou não, podem ser usadas durante os cursos tradicionais de Controle de Processos, em nível de graduação.

Essa necessidade de uso das ferramentas computacionais citadas se justifica, pois o ensino de controle de processos químicos pressupõe a possibilidade da modelagem matemática baseada nos princípios de conservação, complementados com o uso das equações constitutivas do problema, ou o uso dos modelos caixa-preta. 


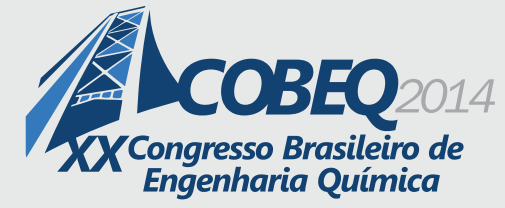

No cotidiano muitas vezes o engenheiro químico é inquirido da necessidade de ressintonizar, ou até mesmo sintonizar, sistemas de controle de processos químicos com modelos desconhecidos, ou parcialmente conhecidos, vindo a necessitar de meios para identificar não só a dinâmica desses processos, mas também para testar o desempenho dos controladores por ele projetados.

Este trabalho teve como objetivos projetar, prototipar, programar e implementar um hardware para emular processos baseados na dinâmica de primeira ordem, operando em malha aberta e/ou malha fechada com um controlador proporcional, integral e derivativo $(\mathrm{P}+\mathrm{I}+\mathrm{D})$, a ser sintonizado. Esse hardware foi construído tendo com base na plataforma de hardware livre microcontrolada Arduino.

Além de satisfazer essa necessidade de primeiro momento, de identificação de uma dinâmica, o mesmo hardware construído, de posse dos parâmetros de sintonia escolhidos pelo usuário, efetiva a simulação em malha fechada com a lei de controle escolhida, permitindo assim a validação do projeto do controlador, sob simulação.

\section{REVISÃO DA LITERATURA}

O campo do controle de processos tem crescido de importância na indústria de processos como consequência da competição global, da volatilidade das condições econômicas e das legislações ambientais e de segurança mais restritas (SEBORG et. al. 2004).

De acordo com Bequette (2003) o ensino de controle de processos químicos nos cursos de graduação de engenharia química é aprimorado com o reforço pelo uso de simuladores computacionais.

A simulação é uma maneira de melhorar a aprendizagem dos estudantes, pois permite aos mesmos se envolverem com aquilo que estão aprendendo de uma perspectiva diferente, propiciando variação no modo de aprendizagem, e incentivando a participação mais ativa no processo de aprendizagem (FRASER, 2013).

Esse uso de simuladores permite atender as necessidades dos cursos de controle de processos químicos, identificadas por Edgar, et. al. (2006), de acordo com as quais os estudantes deverão ser capazes de: desenvolver modelos matemáticos e funções de transferência; analisar a estabilidade de processos e a dinâmica de resposta; determinar empiricamente a dinâmica do processo para a perturbação degrau; entender os diferentes tipos de controladores feedback; analisar e sintonizar controladores PID.

Outras abordagens, que diferem do uso de softwares simuladores, tem sido implementadas. Moor et. al. (2005) desenvolveram kits educacionais para controle de processos. Cada kit custava aproximadamente US\$ 1200, não incluindo o computador pessoal para o sistema de controle. Esses

kits foram desenvolvidos usando a plataforma LEGO® RCX para conversão Analógica/Digital/Analógica (ADA). 


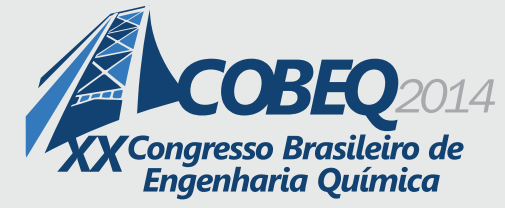

O projeto Arduino foi criado na Itália em 2005 com o objetivo de oferecer uma plataforma de prototipagem eletrônica de baixo custo e de fácil manuseio por qualquer pessoa interessada em criar projetos com objetos e ambientes interativos (PINTO , 2011).

Segundo Monk (2012) o Arduino é uma placa de microcontrolador dotada de conexão USB, que permite a ligação serial com um computador, contendo diversos terminais de conexão com dispositivos externos, como motores, relés, sensores, diodos, etc. O projeto da placa é aberto, configurando o "open hardware", o implica que existe uma variedade grande de placas compatíveis com o projeto aberto Arduino, onde somente a marca Arduino é de uso reservado (BANZI, 2011).

O "open hardware" é aquele projeto de hardware eletrônico que está "disponível gratuitamente sob uma das licenças de código aberto reconhecidas juridicamente" (ACOSTA \& HIPPEL, 2009).

A existência de uma extensa comunidade de usuários da plataforma Arduino, disponibilizando projetos de hardware como "livres", nos moldes do "software livre", acelera o desenvolvimento e a solução de problemas pelos interessados em desenvolver aplicações, sem a formação especializada na área de eletrônica e afins (BANZI,2011)

\section{METODOLOGIA}

Como plataforma de hardware foi usado o microcontrolador Arduino Nano v3.0 (fornecedor Funduino, China), que tem como especificação de hardware: microcontrolador ATmega328, voltagem de operação de $5 \mathrm{~V}$, tensão de alimentação entre 6-9V, 14 pinos digitais de entrada/saída (6 providos de saída modulada por largura de pulso), 8 pinos de entradas analógicas, para corrente elétrica máxima por pino de 40mA, memória flash de 32 KBytes - sendo 30 Kbytes livres para programação, 2 Kbytes de memória SRAM para dados, 1 Kbyte de EEPROM, velocidade de processador de $16 \mathrm{MHz}$, medindo $1,9 \mathrm{~cm}$ por $4,4 \mathrm{~cm}$.

Como ambiente de software de desenvolvimento foi usada a interface Arduino 1.5.4, disponível para download em www.arduino.cc.

Foi implementada na linguagem C++, compatível com o compilador do Atmega328, a solução numérica do problema do valor inicial, pelo método de Euler, da equação diferencial ordinária (1):

$$
\tau . d y(t) / d t=K \cdot x(t)
$$

Os parâmetros constante de tempo, $\tau$, e ganho do processo $\mathrm{K}$, são desconhecidos do usuário e devem ser identificados, através da aplicação do método da curva de resposta dinâmica à perturbação degrau.

A sintonia dos parâmetros do controlador sob a lei $\mathrm{P}+\mathrm{I}+\mathrm{D}$, estruturado conforme a figura 1 , deve ser feita pelo usuário, a partir do tratamento dos resultados da simulação em malha aberta, gerados pelo protótipo construído com o microcontrolador Arduino Nano. 


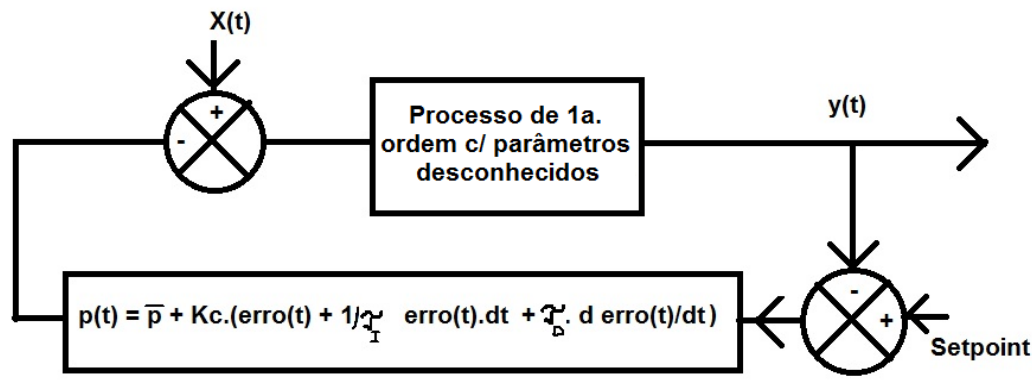

Figura 1 - Malha Fechada do Processo Sob Controle Feedback

$\mathrm{O}$ referido programa baseado na estrutura geral mostrada na Figura 2, permite a entrada e saída de dados através da interface USB, usando-a como interface de entrada/saída.

A necessidade de uso do software de comunicação serial para realizar a interação entre usuário e o ambiente de simulação no microcontrolador representa uma limitação, que é justificada devido aos parcos recursos de hardware do Arduino Nano.

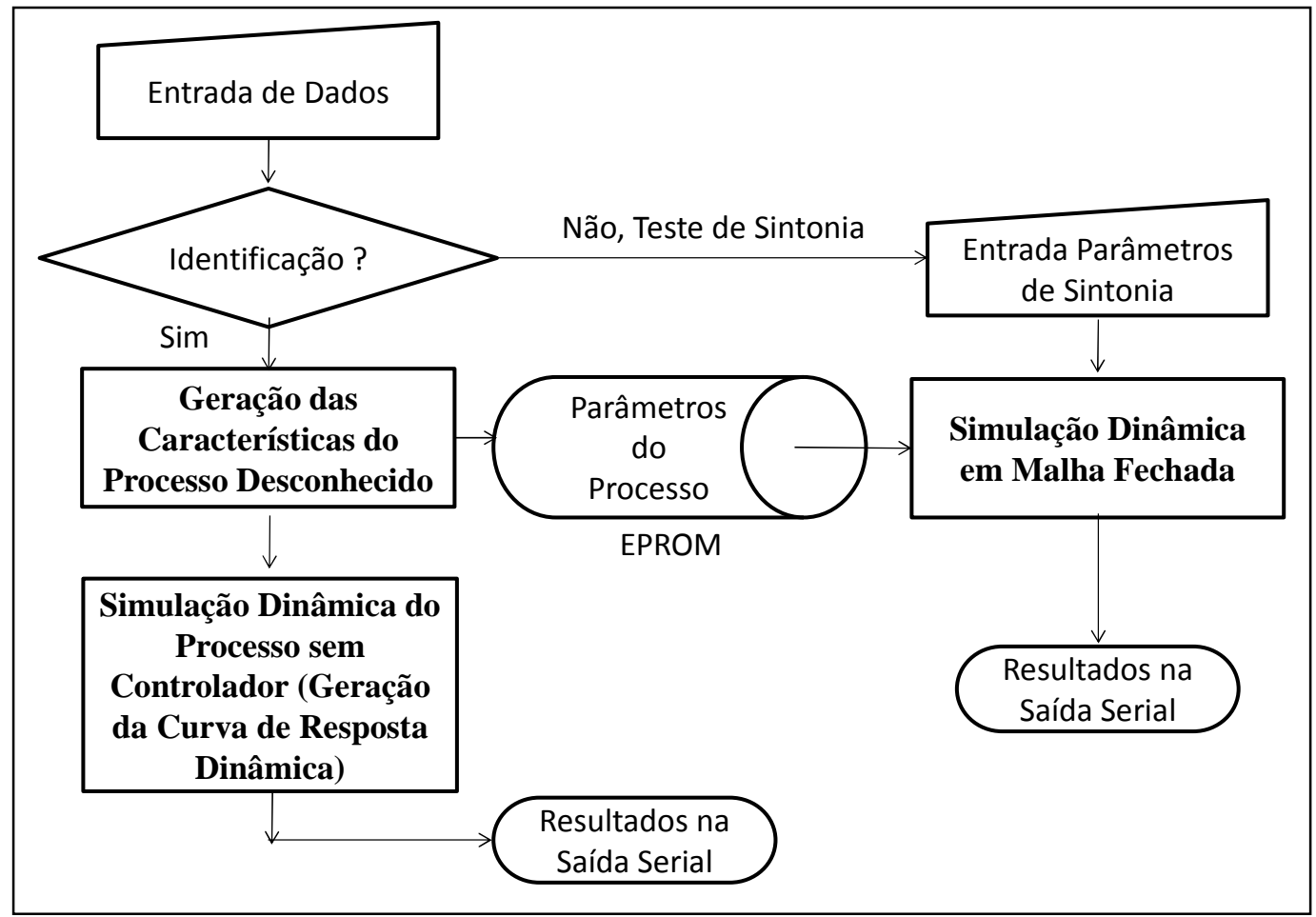

Figura 2 - Estrutura Geral da Ferramenta de Software Implementada no Arduino Nano. 
Há momentos distintos na sistemática de projeto do sistema de controle baseado na arquitetura feedback baseado na lei P+I+D: o momento da identificação dos parâmetros dinâmicos, a sintonia através das heurísticas de Ziegler-Nichols ou de Cohen-Coon, por exemplo, e o teste dos referidos parâmetros ganhos calculados (SEBORG et. al 2004).

A ferramenta de software desenvolvida e incorporada ao microcontrolador Arduino Nano é usada em pelo menos dois momentos. Inicialmente há a geração do comportamento em malha aberta, sob o efeito de uma perturbação degrau na variável manipulada.

Em um segundo momento, definidos os parâmetros do controlador do processo pela heurística de sintonia desejada pelo usuário, é realizada a simulação em malha fechada em estratégia feedback, com a lei de controle escolhida pelo usuário(P, P+I ou P+I+D).

As características ganho do processo e constante de tempo, ficam flutuando ao redor de valores médios, com o uso de números randômicos de forma a possibilitar a geração de um comportamento mais aproximado da realidade, com a presença de ruído na variável manipulada.

Foi utilizada como semente para o gerador de números randômicos a funcionalidade de leitura de uma porta analógica flutuando (sem entrada de sinal) do Arduino, o que aproxima a randomicidade da verdadeira aleatoriedade (MONK,2012).

\section{RESULTADOS E DISCUSSÃO}

Na figura 3 encontra-se a montagem do protótipo com o Arduino Nano, na protoboard, com o display de LCD e a interface USB serial para o computador. O uso de materiais de extremo baixo custo, permite a replicação futura da montagem na forma de kits para o uso.

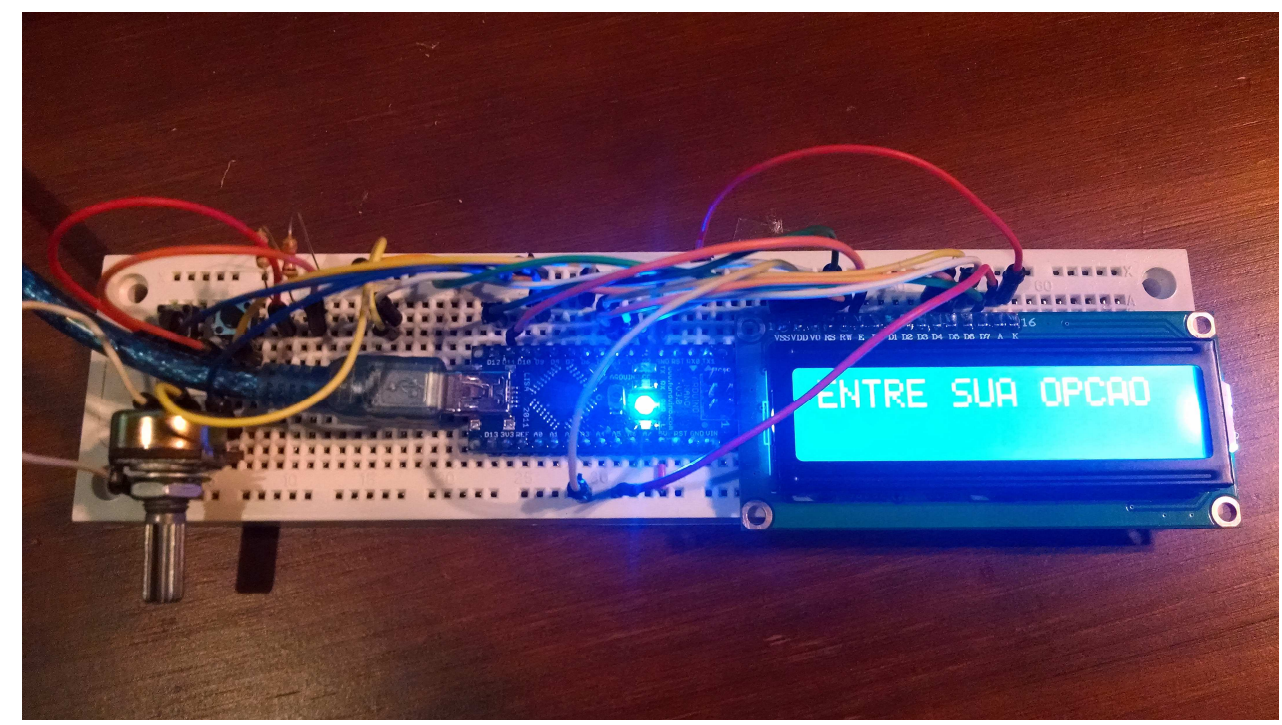

Figura 3 - Montagem do Protótipo Desenvolvido com a Placa Arduino Nano 
Na figura 4 há um exemplo de curva de resposta dinâmica, obtida a partir de simulação no Arduino Nano, confeccionada com a planilha Calc do LibreOffice, após o tratamento dos resultados transferidos no formato de valores separados por vírgulas (CSV).

A simulação computacional e a consequente curva de resposta dinâmica da figura 4 , com o conhecimento da amplitude do degrau utilizado na perturbação, permitiriam a identificação dos parâmetros ganho do processo e constante de tempo, para a identificação do processo desconhecido.

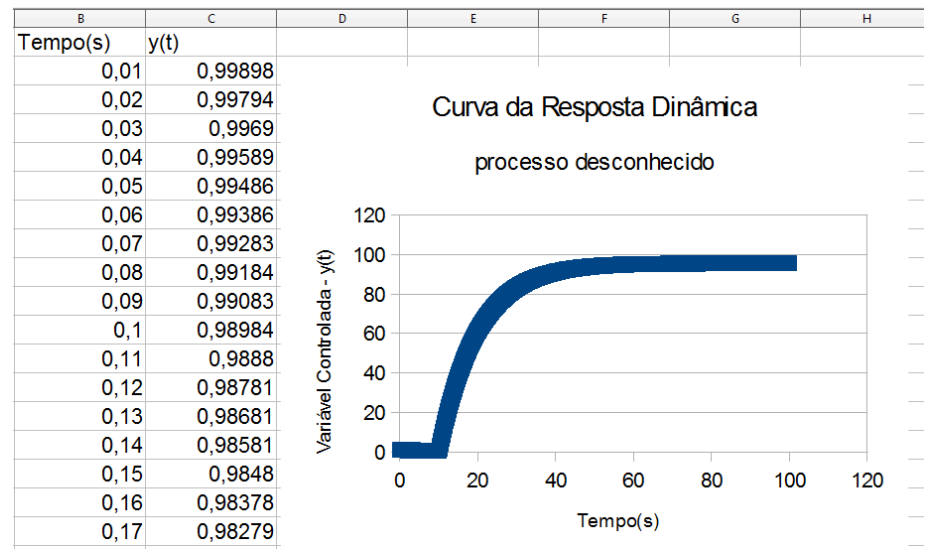

Figura 4 - Exemplo de Resposta Dinâmica Confeccionada a partir dos Resultados de Simulação no Arduino Nano

Depois de usada a sintonia pelo método de Cohen-Coon (SEBORG et. al., 2004), em malha aberta, os resultados do desempenho encontram-se na figura 6 . Todo o processamento de simulação do sistema desconhecido, em malha aberta ou fechada é feito no Arduino Nano.

\section{Resposta da Variável Controlada}

Durante Sintonia

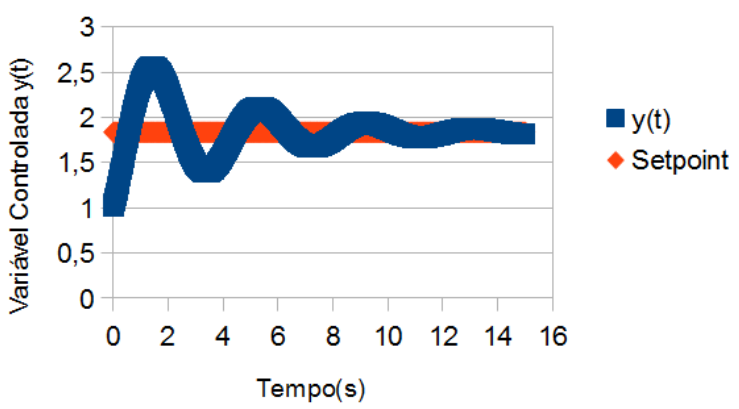

Figura 5 - Exemplo de Comportamento da Variável Controlada Durante Sintonia Realizada com o Simulador no Arduino Nano 


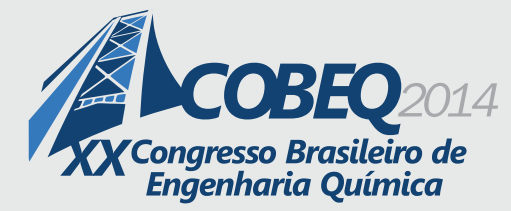

Algumas das dificuldades apresentadas pelos estudantes durante o uso do protótipo desenvolvido foram a estranheza com plataforma, e a dificuldade em configurar o driver da porta serial no sistema operacional do microcomputador usado, sendo que inclusive alguns estudantes sobregravaram um programa diferente na memória do Arduino Nano, perdendo o programa original.

Por outro lado, a curiosidade e o conhecimento do uso de uma ferramenta de hardware de microeletrônica, baseada em microcontrolador, foi desafiador e serviu como estímulo em alguns dos relatos dos estudantes da disciplina de Controle de Processos Químicos do curso de Engenharia Química da Universidade Federal do Rio Grande - FURG.

\section{CONCLUSÃO}

Foi montado um protótipo, usando a plataforma de hardware livre Arduino, capaz de emular processos químicos baseados na dinâmica de primeira ordem, operando em malha aberta e/ou malha fechada com um controlador $\mathrm{P}+\mathrm{I}+\mathrm{D}$.

A iteratividade do usuário com o referido com o protótipo construído é feita através da interface serial de um computador pessoal, tanto para a entrada quanto para a saída de dados. As simulações dinâmicas são executadas inteiramente no microprocessador ATmega328 do Arduino Nano utilizado.

O protótipo de equipamento desenvolvido se mostrou uma ferramenta útil no ensino de identificação de parâmetros de processos dinâmicos e na sintonia de controladores feedback com lei $\mathrm{P}+\mathrm{I}+\mathrm{D}$, para o curso de Engenharia Química da Universidade Federal do Rio Grande.

As principais vantagens do protótipo desenvolvido estão associadas com o seu baixo custo de construção, com a facilidade de replicação do projeto, com a inserção do uso de microcontroladores no âmbito da disciplina de Controle de Processos Químicos.

As eventuais desvantagens e dificuldades do uso do protótipo relatadas durante a aplicação estiveram associadas com o desconhecimento do funcionamento da plataforma microcontrolada Arduino, em especial quanto à sua arquitetura, e à restrita capacidade de instalar corretamente os drivers de portas seriais no sistema operacional por parte dos estudantes da disciplina.

Será objeto de desenvolvimento futuro a aplicação de microcontroladores no controle e automação de processos químicos, baseados especificamente no uso do "hardware livre" em malha fechada, com técnicas tradicionais ou não de controle de processos, através do uso da plataforma Arduino. 


\section{REFERÊNCIAS}

ACOSTA, R.; HIPPEL, E.; Open Source Hardware, MIT, 2009.

BANZI, M.; Getting Started with Arduino. Maker Media, Inc, 2011.

BEQUETTE, B.W.; Process Control: Modeling, Design and Simulation, Prentice Hall, (2003).

FRASER, D.M.; Teaching Chemical Engineering using Computer Simulations, Proceedings of the 6th International Conference on Process Systems Engineering (PSE ASIA), Kuala Lumpur, 2013.

EDGAR, T. F.; OGUNNAIKE, B. A.; DOWNS, J. J.; MUSKE, K.R.; BEQUETTE, B.W.; Renovating the undergraduate process control course, Computers \& Chemical Engineering Vol. 30 10-12, 12 pp.s 1749-1762, 2006.

MONK, S.; Programming Arduino Getting Started with Sketches. McGraw-Hill, 2011.

MOOR, S.S.; PERGIOVANNI, P.R.; METZGER; M.; Process Control Kits: A Hardware and Software Resource, 35th ASEE/IEEE Frontiers in Education Conference

PINTO, M. de C.; Aplicação de Arquitetura Pedagógica Em Curso de Robótica Educacional com Hardware Livre. Rio de Janeiro: UFRJ, 2011. 158 f.: Il.

SEBORG, D.E., EDGAR, T.F.; MELLICHAMP, D.A. ; Process Dynamics and Control, 2a Ed., Wiley. 2004. 